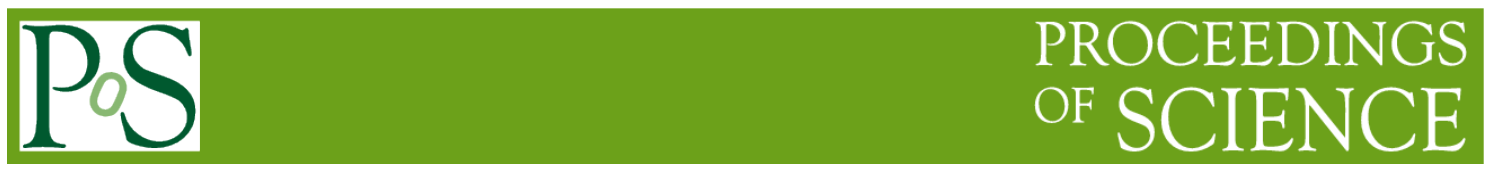

\title{
Application of CAUX in Remote Evaluation of Websites
}

\author{
Ziwei Wang1 \\ Dalian Maritime University \\ Dalian, 116026, China \\ E-mail: 944084267 @qq. com

\section{Zhengjie Liu} \\ Dalian Maritime University \\ Dalian, 116026, China \\ E-mail: Iiuzhj@dlmu.edu.cn
}

\begin{abstract}
In order to address problems of recording data in a complex way and utilizing unreal scene in traditional methods, the author proposes a method about remote evaluation of websites by using the Context-Awareness User Experience tool. This method helps researchers get the user data from real environment. The CAUX tool can collect user data automatically without disturbance, and it can also make semi-automated analysis of the data to reduce the burden on UX researchers. The CAUX tool needs to be installed in the user's mobile phone in advance. It can collect data of the situation about users according to the collection rules set by the UX researcher. The user data, which include objective data, URL sequences, screenshot etc, can be uploaded to the server database automatically for storage. These data can also be presented in a visual interface. Then UX researchers analyse them to figure out usability problems. In this paper, the author applies the CAUX tool to a case to study the feasibility of the remote user experience evaluation method. The case is selected as the website of Dalian maritime university. The results indicate that the application of CAUX tool in analysing user experience about remote mobile site can be more realistic and objective. Also, the data are shown in a form of visualization so as to improve UX researchers data analysis efficiency and help UX researchers conduct user experience research more effectively.
\end{abstract}

\author{
ISCC2017 \\ 16-17 December 2017 \\ Guangzhou, China
}

\section{${ }^{1}$ Speaker}




\section{Introduction}

With the popularity of the smart phones, billions of users have acquired an easy access to visit websites. The competition among websites is more and more intense. User experience (UX) is one of the most important factors to determine whether users will continue to use the websites [1]. Therefore, Websites evaluation can improve the competitiveness of websites, and bring huge benefits at the same time.

The traditional user experience evaluation is carried out in the laboratory where the professional equipment can support evaluation; however, it suffers from the disadvantage of being costly and time consuming[2]. This has driven businesses and academics to consider remote usability evaluation as an "increasingly important alternative to conventional usability evaluation"[3].

Hartson HR et al proposed the conception of remote evaluation in 1996[4]. Remote evaluation allows users to perform tasks in natural environment. This method can obtain the experience of users in the natural situation which meets users' usual habits. Based on the remote evaluation, the author proposes a new method for remote evaluation of websites by using the Context-awareness user experience (CAUX) tool. This method can collect users' data automatically and analyze then in a semi-automated way. In the second chapter, the author introduces the CAUX tool and describes general overview of the whole study. The third chapter introduces how to enhance the capabilities of CAUX tool in remote evaluation of websites. The fourth chapter briefly describes the entire experiment process, including experimental implementation, experimental analysis and results. The fifth chapter summarizes the whole study, covering its advantages, deficiencies of CAUX in the remote evaluation of websites, and lastly forecasts the future development direction.

\section{Overview}

\subsection{User Experience Research Tools}

Past attempts of automation have also resulted in tools that are too specific and application- related, but very costly and oversimplified[5], such as TEA[6], which can only collect user data. They can not analyze user data. With the deepening research, the semiautomatic data analysis function has also been gradually applied to the remote user experience research tools such like MATE[7]. However, these research tools are still in the development stage, and they still have some shortcomings:

1). User's behaviour is contextual. But these remote user experience research tools do not consider the coupling between context and user behaviour.

2) Accurate and inaccurate data collection is not accurate and timely for situation information.

The CAUX tool is used for researching user experience. It combines context-aware models with remote research techniques. It adopts mobile devices to capture subjective and objective data about users. CAUX tool includes client and server. Users run the client on mobile devices, CAUX collect user data and upload data within the specified time automatically. UX Researchers can log onto the server to download the users' data. However, the current CAUX tool is still in groping phase. It is necessary to improve its capabilities to help UX researchers evaluate user experience remotely. 


\subsection{Study Overview}

The author studies CAUX tool through iterative method. In the early stage, the tool extends through literature research. Then the author uses CAUX tool to do some case studies which can extend and modify CAUX tool according to continuous case study so that it can better help UX researchers evaluate UX remotely. The author evaluates 20 websites including shopping sites, information portal sites, travel sites and others in order to ensure the universality of CAUX. In the research, the author summarizes how to use CAUX tool in remote evaluation of websites. Then the author uses CAUX tool to do case studies, summarize case studies and propose usability problems.

\section{Extend CAUX Tool Capabilities}

According to literature research and case studies, CAUX tool should be extended in the following two aspects.

\subsection{Get the URL and Time}

Traditional browser applications do not provide the exact browsing time, which make UX researchers unable to access quantitative data thereby influencing the analysis of user experience on websites. The initial version of CAUX tool can collect data including APP status, users context information and device information with the data collected shown in Figure 1. But it could not provide the ability to browse websites. Therefore, the author expands the CAUX tools based on the existing CAUX, and adds Website browsing capability by using the Webview control. The expanded tool can collect data including app status, context information and device information; besides, it can collect website operation information including URL and visit time. The modified users' data are shown in Figure 2.

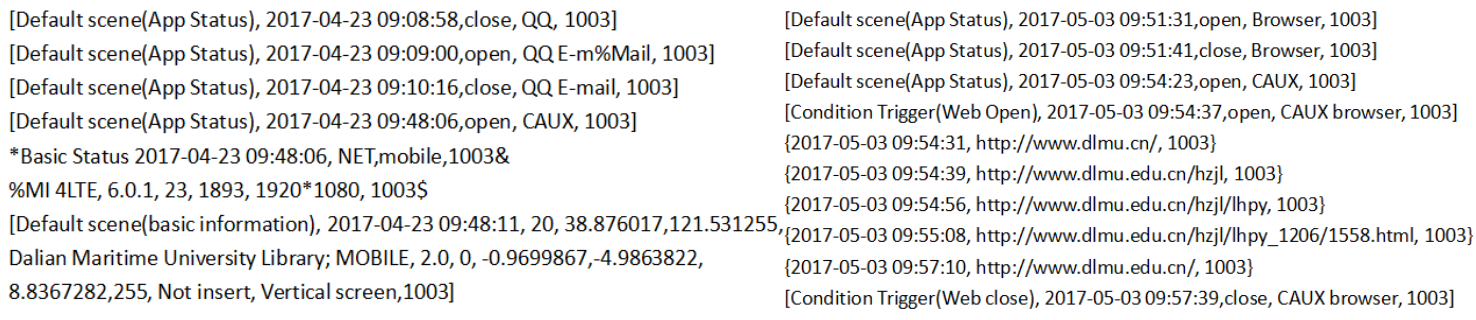

Figure 1: Original Users data

Figure 2: Modified Users data

\subsection{Data Visualization}

To reduce the data analysis burden on UX researchers, the author adds the capability of semi-automatically data analysis and data visualization to CAUX tool. The data analysis and data visualization need remote evaluation of website: 1). Statistics: including user completion rate, direct completion rate, path key rate, time of users to complete tasks and so on. 2), Websites information architecture.

According to the above needs, the data visualization should be divided into three modules, which include data setting module, data showing module and data editing module. The data setting module can select user and time. The data showing module includes website information architecture, statistics, device information and context information of users. UX researchers can mark, remark and edit the visual data by data editing module. The author uses Echarts in 
combination with web technology to implement data visualization. It includes user's number, context information, device information, app status, subjective data and user's URL path.

\section{Application of CAUX Tools in Remote Evaluation of Websites}

\subsection{Evaluation Implementation}

On the basis of website of Dalian University of Foreign Languages, in the early stage, the author analysed the target user groups of Dalian University of Foreign Languages website and obtained the main tasks of the target groups in this website. Then the author recruited users for the remote evaluation. After the users have completed the tasks, UX researchers collected and analysed the data. Finally, they found out problems about Dalian University of Foreign Languages and give suggestions for improvement.

\subsubsection{User Recruitment}

In 2016, Janet M. Six and Ritch Macefield concluded that 8-10 people shall be the best population for evaluation [8]. So the author recruited 10 people for this evaluation. The recruitment proceeded online mainly through $\mathrm{QQ}$, WeChat, forums and others.

\subsubsection{Evaluation Task}

After understanding the college students' browsing behaviour on the website of Dalian University of Foreign Languages, the author proposes four evaluation tasks to evaluate user experience about the website of Dalian University of Foreign Languages. During the evaluation, all participants perform the same tasks because the task assignment is reported to have a significant effect on usability evaluation [9]. The tasks are shown as follows: 1. Please find the webpage which introduces the student union of software Institute.

2. Please find the name of the secretary of current party committee secretary.

3. Please find the Office of Academic Affairs contact information.

4. Please find instructions for studying abroad.

Users can perform tasks anytime and anywhere. When users open the CAUX browser, the tool records users' quotations automatically. CAUX tool can also automatically get user's screen information when they perform tasks. Those data can help UX researchers for later data analysis.

\subsubsection{Rules Setting}

Using CAUX tool in remote evaluation can automatically record user data, before which some rules for data collection are required. The form of rule setting is "IF (condition) THEN (operation)". The rule setting of this evaluation is shown in Table 1.

\begin{tabular}{|l|l|}
\hline IF & THEN \\
\hline CAUX open & Time, location, battery, network, URL \\
\hline CAUX close & Time, location, battery, network, URL \\
\hline CAUX browser open & Time, location, battery, network, URL, recording, screenshot \\
\hline CAUX browser close & Time, location, battery, network, URL, recording, screenshot \\
\hline Screen open & Time, location, battery, network, URL \\
\hline Screen off & Time, location, battery, network, URL \\
\hline
\end{tabular}

Table 1: Rules Setting 


\subsection{Data Analysis}

The author choose task 1 as an example to illustrate the whole analysis process. Firstly, he restores user scenario based on the visual information of the user. Details are shown as Figure 3. The scenario is the user named 1003 who performs the task in Dalian Foreign Studies University Library at 9:56 am. The network is WIFI. The phone's battery is $75 \%$, and the screen brightness is 87 , and the light is sufficient.

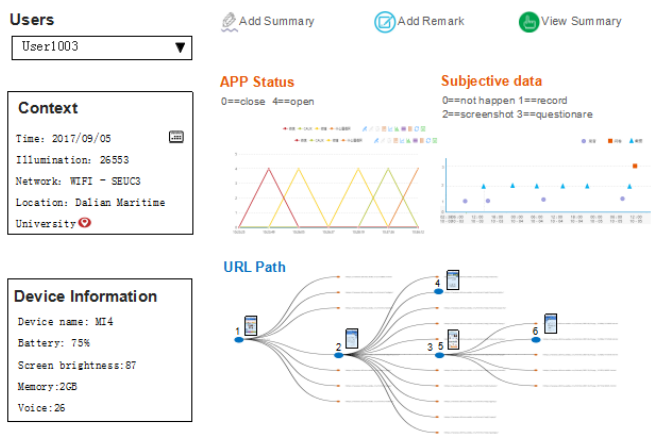

Figure 3 : Visual Information

Figure 4 is the comparison between the expected sequence and the user's actual sequence. As we can see from the visualization, the expected sequence of task 1 is 4 steps, but the actual steps of the user are 6. By comparing the two operation sequences, it can be easily found that the user operates correctly in the previous three steps, but error occurred in the fourth step. Finally, the user returns to the correct operation after two consecutive errors. When the data is restored in the websites it can be known that error occur after the user enters the "Learning Organization" module. The user do not successfully find the "student union" in "Learning Organization" module, the user enters the "student activities" module, which indicating that the user does not pay attention to the student union information on the right side. In the user's recording during the task, the user says "why there is no feedback when I click?" In this step, the user focuses on the menu located in the left side, and do not pay attention to the change of the right side. Just because there is no feedback when the user clicks the "Student Organization", the user clicks another button. Therefore, it should give feedbacks to users when they click the button on the left menu.

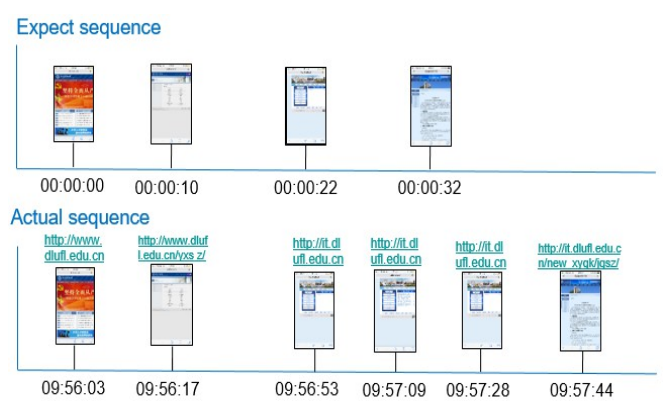

Figure 4: Sequence Comparison

\subsection{Results}

This evaluation recruits 10 users, collects 6704 pieces of data information including 317 screenshots, 6355 text messages, 32 recordings in five days. After analysing the data, the author finds seven usability problems, as shown in Table 2. 


\begin{tabular}{|c|c|c|}
\hline & Usability Problems & Comments \\
\hline 1 & $\begin{array}{l}\text { The left navigation bar under the "Learning } \\
\text { Organization" module has no click feedback } \\
\text { when clicked, so users often click repeatedly } \\
\text { and click other buttons when get no reaction }\end{array}$ & $\begin{array}{l}\text { The left navigation bar under " } \\
\text { Learning Organization " provides } \\
\text { feedback. After clicking the navigation } \\
\text { bar it should display the classification } \\
\text { of the module }\end{array}$ \\
\hline 2 & $\begin{array}{l}\text { The percentage of users visiting the "Student } \\
\text { Affair Offices" interface reached } 100 \% \text {, which } \\
\text { means that most users think the student union } \\
\text { belongs to the "Student Affair Offices" module }\end{array}$ & $\begin{array}{l}\text { Modify the "Student Affair Offices" } \\
\text { into a "Student Party Committee } \\
\text { Office" to avoid confusion }\end{array}$ \\
\hline 3 & $\begin{array}{l}\text { Navigation bar does not have sub navigation } \\
\text { content, users could not know contents about } \\
\text { the module }\end{array}$ & $\begin{array}{l}\text { Add that first click trigger displaying } \\
\text { sub-navigation content, twice click } \\
\text { enter the link }\end{array}$ \\
\hline 4 & $\begin{array}{l}\text { There is no detailed information in the teachers' } \\
\text { faculties in each college, and the user cannot } \\
\text { find teachers' information }\end{array}$ & Add teachers' information \\
\hline 5 & $\begin{array}{l}\text { Click "contact us" in the bottom of the home } \\
\text { page but no feedback }\end{array}$ & Add "contact us" interface \\
\hline 6 & $\begin{array}{l}\text { Notification at the "International Exchange" is } \\
\text { displayed in a scroll bar. However, there are no } \\
\text { signs about the quantity of notifications, so } \\
\text { users can only wait to see all }\end{array}$ & $\begin{array}{l}\text { Add sign to show the number of } \\
\text { announcement, and shortcut menu, so } \\
\text { that users can see any notification } \\
\text { quickly }\end{array}$ \\
\hline 7 & $\begin{array}{l}\text { "Study Abroad" is a long-term journey, but } \\
\text { "International Exchange" is not. And in the } \\
\text { web, "Study Abroad" is belong to } \\
\text { "International Exchange". So it is incorrect. }\end{array}$ & $\begin{array}{l}\text { Separate "Study Abroad" from the } \\
\text { "International Exchange" module. Set it } \\
\text { as a single module }\end{array}$ \\
\hline
\end{tabular}

Table 2: Results of the Case Study

After users have finished the tasks and exited the CAUX browser, the tool automatically pops up a questionnaire. This questionnaire is SUS standard fractional scale [10], which evaluates the website satisfaction. The scores are divided into several stages, "0-50" is F, 50-60 "for E ..." 90-100 "for A. According to the questionnaire, the user's satisfaction degree is 72.5, which is at the $\mathrm{C}$ level, indicating that the website need better user experience.

\section{Conclusion}

Based on situational awareness, the author proposes a method for remote evaluation of websites. This method uses CAUX tool to collect user data automatically and analyse the data semi-automatically. The author explores the capabilities that CAUX tools should have in iterative experiments. To reduce the data analysis burden on UX researchers, the author proposes a semi-automated data analysis method. Then the author applies the expanded CAUX tool to do some case studies for verification. Experiments show that this method can obtain real operation data from the users under the real environment to analyse the real user experience when using the website and making up for the gaps in the evaluation of CAUX tool. Experiments show that compared with traditional methods, using CAUX to do remote evaluation has following advantages: 1 . The users do not need to do the evaluation at the professional lab. They can be in the real environment. 2 . Some usability problems are related to the context. The traditional methods cannot collect context information. But CAUX tool can get user date with contextual information. 3. Instead of getting user data manually in traditional method, CAUX tool can get data automatically. User data is more precise. 4. Traditional methods cannot provide visual data, while the CAUX tool can, and it reduces burden on UX researcher. 
The application of CAUX tool in remote evaluation of websites has achieved the expected goals. Due to time and resource constraints, some of the research still need to be improved. Semi-automatic data analysis method cannot automatically identify the UX problem. UX researchers are still burdened by calculating the huge amount of user data. In the future, the author will continue to explore the tools' data analysis capabilities.

\section{References}

[1]Marc Hassenzahl, Noam Tractinsky. User experience - a research agenda[J]. Behaviour \& Information Technology, 25(2):91-97(2006).

[2]Bastien J M. Usability testing: a review of some methodological and technical aspects of the method $[\mathrm{J}]$. International Journal of Medical Informatics, 79(4):e18(2010).

[3]Andreasen M S, Nielsen H V, Stage J. What happened to remote usability testing?:an empirical study of three methods[C]. Sigchi Conference on Human Factors in Computing Systems. ACM,:1405-1414(2007).

[4]Hartson H R, Kelso J, Neale W C. Remote evaluation:the network as an extension of the usability laboratory $[\mathrm{C}]$. Conference on Human Factors in Computing Systems: Common Ground, CHI '96, Vancouver, Bc, Canada, April 13-18, 1996, Proceedings. DBLP:228-235(1996).

[5]Mifsud J, Dingli A. USEFul: A Framework to Mainstream Web Site Usability through Automated Evaluation[J]. International Journal of Human-Computer Interaction:2011-2010(2011).

[6]Obendorf H, Weinreich H, Hass T. Automatic support for web user studies with SCONE and $T E A[C]$. CHI '04 Extended Abstracts on Human Factors in Computing Systems. ACM:1135$1138(2004)$.

[7]orat T, Schclar A, Shapira B. MATE: a mobile analysis tool for usability experts[C]. CHI '13 Extended Abstracts on Human Factors in Computing Systems. ACM:265-270(2013).

[8]Janet M. Six, Ritch Macefield. How to Determine the Right Number of Participants for Usability Studies. UX matters( 2016).

[1]Bruun A, Stage J. The effect of task assignments and instruction types on remote asynchronous usability testing $[\mathrm{J}]: 2117-2126(2012)$.

[9]Bangor A, Kortum P, Miller J. Determining what individual SUS scores mean: adding an adjective rating scale $[\mathrm{J}]$. Journal of Usability Studies, 4(3):114-123(2009). 